\title{
Los principios de autotelia y de no arbitrariedad en el concepto de ideología usado por Francisco Miró Quesada Cantuarias
}

\author{
THE PRINCIPLES OF AUTOTELIA AND NON-ARBITRARINESS \\ IN THE CONCEPT OF IDEOLOGY USED BY FRANCISCO \\ MIRÓ QUESADA CANTUARIAS
}

Javier Ulises Aldama Pinedo

Universidad de Lima

jaldama@ulima.edu.pe

\section{RESUMEN}

El planteamiento ideológico del filósofo peruano Francisco Miró Quesada Cantuarias es humanista, racionalista, progresista y occidentalista. Estas características se mantienen a lo largo de su obra escrita; sin embargo, se presenta un cambio de perspectiva relacionado con los principios en los que se sostiene su propuesta. En un primer momento, clasifica a su ideología como estimativa, con relación a uno de los principios que postula: el principio de autotelia, el cual simplemente se asume como un acto de fe y de acción; el otro principio que presenta es el principio de no arbitrariedad, que se vincula a la crítica racional. En un segundo momento, el principio de autotelia se replantea como proyecto, y a su vez destaca la importancia del principio de no arbitrariedad como condición necesaria y suficiente en el campo de la praxis. Finalmente, plantea el principio de simetría como complemento del principio de no arbitrariedad.

PALABRAS CLAVE: principio de autotelia, principio de no arbitrariedad, principio de simetría, ideología, praxis

\section{ABSTRACT}

The ideological approach of Peruvian philosopher Francisco Miró Quesada Cantuarias is humanist, rationalist, progressive and occidentalist. He maintains these characteristics throughout his written work; however, there is a change of perspective related to the principles on which his proposal is based. At first, he classifies his ideology as estimative, relating it to one of the principles he postulates: the autotelic, assumed as an act of faith and action; the other principle he presents is non-arbitrariness, which is linked to rational criticism. In a second moment, the autotelic principe is restated as a project, in turn emphasizing the importance of the principle of non-arbitrariness as a necessary and sufficient condition in the field of praxis. Finally, he postulates the principle of symmetry as a complement to non-arbitrariness.

\section{KEYWORDS: principle of autotelia, principle of} non-arbitrariness, principle of symmetry, ideology, praxis 


\section{INTRODUCCIÓN}

El tema ideológico es un asunto del que se ocupó Francisco Miró Quesada Cantuarias (FMQC) en diferentes ensayos. Para los dos principios que abordaremos, son de especial importancia "La ideología humanista" (1965) y "El intelectual, el Occidente y la política" (1965), ambos ensayos publicados respectivamente como los capítulos III y IV del libro Humanismo y revolución (1969). En la tercera parte de una obra anterior, Manual ideológico. Primera parte (1967), se presenta "El concepto de ideología", escrito entre 1965 y 1966, y que aparece como el capítulo I de Humanismo y revolución, texto importante para esclarecer lo que es ideología para FMQC y su clasificación, del cual tomaremos las citas en este artículo.

Otros trabajos que tenemos en cuenta son "Filosofía, ideología y revolución" (1990) y "Los nuevos valores de la cultura contemporánea y su proyección en el futuro de la humanidad" (1980); estos dos ensayos fueron publicados en Hombre, sociedad y política (1992). Igualmente consideramos, del libro Ser humano, naturaleza, historia (2003a), el ensayo homónimo para esta edición, "Ser humano, naturaleza, historia" (su primer título, publicado en 1987, fue "Hombre, naturaleza, historia"), y el "Ensayo de una fundamentación racional de la ética" (2003); este trabajo es señalado por FMQC (según la anotación que hace David Sobrevilla en el prólogo del libro) como "la culminación de toda su trayectoria filosófica" (Miró Quesada, 2003a, p. 10). Finalmente, hemos considerado el capítulo III de la primera parte de El hombre, el mundo, el destino, titulado "Introducción no convencional a la filosofía" (2003b).

La importancia de la propuesta de FMQC y su peculiaridad es el rescate, nuevamente, de lo racional para la práctica política; otras propuestas ideológicas también se proclaman humanistas, pero destacan como aspectos principales el factor económico, cultural, étnico o de género. Esta diferencia (que es notoria en su obra) es la razón por la que FMQC incide en los principios que aquí abordamos.

\section{EL SENTIDO DE LA PALABRA IDEOLOGÍA, SEGÚN FRANCISCO MIRÓ QUESADA CANTUARIAS, Y SU CLASIFICACIÓN}

Cuando se aborda el tema ideológico, es necesario indicar cuál es el sentido en que se emplea el término ideología, debido a los diferentes usos que se hace de esta palabra. Miró Quesada (1969), al respecto, es preciso: la ideología es "la fundamentación racional de la praxis política" (p. 20). Esto no significa que solo la filosofía pueda fundamentar la praxis política, pues también pueden cumplir con este papel "la ciencia, la religión, el mito, etcétera" (p. 26).

Es evidente, además, que hay diferentes ideologías, Miró Quesada (1969) propone al respecto la siguiente clasificación: (1) las ideologías epistémicas o teóricas, "que basan 
la aceptación de sus principios en la pretensión de que son verdades filosóficas o científicas" (p. 28); y (2) las ideologías estimativas o timéticas, que "fundan la pretensión de realización de sus principios en estimaciones de valor, en creencias, en dogmas religiosos, o simplemente en la voluntad colectiva" (pp. 28-29).

La mayoría de las grandes ideologías occidentales son del primer tipo, por ejemplo, la ideología enciclopedista, la burguesa-liberal, el marxismo, etcétera; del segundo tipo son las ideologías socialcristianas y la mayor parte de las ideologías humanistas. En este último tipo de ideología no se usan proposiciones que pretenden describir hechos - como en las ideologías epistémicas - y que luego de someterse a verificación pueden ser verdaderas o falsas, sino que se proclaman normas, "actos de voluntad que indican cómo deben comportarse los hombres" (Miró Quesada, 1969, p. 31).

FMQC opta por asumir una ideología estimativa, aunque pueda parecer paradójico que un pensador racionalista y con una sólida formación científica como él adopte esta posición. Aparte de la amplia explicación que brinda y que veremos de manera resumida luego, consideramos que lo que le lleva a esta posición es una especie de aplicación del criterio de falsación de Popper (1980), que se basa en un juicio de valor y preferencia (p. 37), pero usado en el ámbito ideológico.

Debemos advertir también que el origen del planteamiento ideológico lo ve Miró Quesada (2003a) en el Renacimiento y hace hincapié en Francis Bacon (p. 16) como el primer cuestionador de la inautenticidad de las clases dominantes y quien, a su vez, postula una nueva sociedad; sin embargo, considera que "su pensamiento no es aún suficientemente sistemático" (Miró Quesada, 1969, p. 49), de modo que lo ubica junto a Hobbes en una etapa preideológica. En cambio, Locke, Hume y los principios del racionalismo y el empirismo de los siglos XVII y XVIII hacen posible que con los ilustrados surja “la primera ideología de la historia” (Miró Quesada, 1969, p. 50).

La ideología enciclopedista y sus planteamientos son algo con lo que -en generalla propuesta de Miró Quesada (1969) se identifica. A las ideologías de los siglos XVIII y $\mathrm{XIX}$ las califica como especulativas, porque considera que carecen de rigor y que buscan resultados amplios. Estas ideologías, aunque no han desaparecido, han sido superadas - desde su punto de vista - por las ideologías axiomáticas del siglo Xx; estas "muestran que toda fundamentación de la praxis para ser rigurosa debe partir de axiomas o postulados bien definidos" (p. 57).

\section{EL HUMANISMO EN EL PLANTEAMIENTO IDEOLÓGICO DE FRANCISCO MIRÓ QUESADA CANTUARIAS}

Al calificar su propuesta de humanista, FMQC se refiere a la afirmación del valor incondicional de la condición humana y esto a su vez nos remite, sobre todo, al hombre como 
un fin en sí mismo: "El humanismo consiste en considerar que la persona es un fin en sí mismo, y que la sociedad debe organizarse de manera que el individuo pueda realizar sus máximas potencialidades" (Miró Quesada, 2003b, p. 49).

Como ideología, esto lleva a la búsqueda de la liberación del ser humano de toda estructura u orden que lo convierta en un medio, por lo que es contraria a la clásica división entre la élite y el pueblo o entre dominadores y dominados. Este tipo de propuesta, advierte, solo llega a la madurez a fines del siglo XVIII y comienzos del XIX en la civilización occidental, y responde a dos raíces de esta: el cristianismo y el racionalismo. Del primero se considera su concepción del hombre como individuo, la liberación del hombre real y la afirmación del valor de los pobres; del segundo, su análisis que convierte a la verdad en valor absoluto y que funciona en el ámbito práctico como una especie de disolvente de los criterios que usaban los grupos dominantes para sostener su primacía. (Miró Quesada, 1969, pp. 92-100; 1992, pp. 104-110).

Asumir el humanismo, advierte Miró Quesada (1992), lleva hacia un determinado tipo de sociedad:

En la sociedad racional o humanista, cada ser humano puede vivir libremente y vivir libremente es asumir los valores que más significación tengan para nuestra vida. En una sociedad semejante, no hay tablas fijas de valores, no hay modelos de conducta que se deban seguir obligatoriamente, so pena de sufrir graves sanciones. (p. 210)

Es Kant quien formula el principio que considera al hombre como un fin en sí mismo:

[Y] lo que solo puede ser fin en sí mismo y nunca medio no tiene precio, porque no es cosa, sino persona y tiene por eso dignidad; llamamos a este principio, formulado de manera definitiva por Kant, principio de la autotelia. (Miró Quesada, 1969, p. 98)

\section{EL PRINCIPIO DE AUTOTELIA}

Es un principio que, como señala FMCQ, se remonta a una de las fórmulas planteadas por Kant del imperativo categórico: "Obra de tal modo que uses la humanidad, tanto en tu persona como en la persona de cualquier otro, siempre como un fin al mismo tiempo y nunca solamente como un medio" (Kant, 1980, pp. 44-45).

Miró Quesada (1969) presenta el principio de autotelia como uno de acción que asume el hombre y cuyo fin es el mismo hombre; se trata también de una actitud humanista. Pero, además, considera que "es suficiente para que el humanismo quede constituido. Todos los demás principios se pueden deducir de él por medio de la lógica y del análisis semántico" (p. 102). Así, son deducibles el principio de solidaridad y el antiimperialismo; con respecto a la solidaridad, nos muestra que el individuo no es un ente solitario, sino 
que es alguien en relación de interdependencia y realización con los otros. Pero, aun en esta relación, tiene prelatura el individuo y esto a fin de que no sea subordinado por el colectivo. La acción generada por el principio de autotelia se dirige hacia la sociedad justa o sociedad sin clases, que es básicamente un ideal y el único medio para llegar a ella es la democracia:

\begin{abstract}
Una sociedad justa debe ser también libre. En efecto, si se parte del principio fundamental del humanismo, es decir, del principio autotélico, se tiene que llegar necesariamente a la conclusión de que la sociedad debe organizarse democráticamente. Si se acepta la dictadura, se acepta la violencia, y si se acepta la violencia, se reduce a los que no ejercen el poder a la condición de medios o instrumentos. (Miró Quesada, 1969, p. 116)
\end{abstract}

Desde ya Miró Quesada considera la ideología humanista como revolucionaria, pues pretende cambiar las estructuras de dominación y de situación de privilegio de las élites, y radical, ya que busca llevar hasta las últimas consecuencias los principios que asume. Asimismo, esta ideología es opuesta al uso de la violencia, a los que predican lo ineluctable de esta para generar un cambio efectivo en la sociedad. Presenta el ejemplo de la revolución nórdica y la transformación llevada a cabo en países como Canadá o Nueva Zelanda.

Importante es notar que Miró Quesada (1969) señala la forma de identificar al hombre como fin en la acción de la siguiente manera: "Lo único que permite saber cuándo un hombre es un fin en sí es su capacidad de decisión" (p. 119); es decir que el hombre es libre en cuanto dispone de autonomía para decidir y actuar. Otra expresión de esta idea es la siguiente:

Los principios ideológicos del humanismo como el principio autotélico [...], no se funda en teorías de ninguna clase, sino en una expresión de la voluntad de los oprimidos de afirmarse frente a sus opresores como hombres libres y dueños de sus destinos. (p. 29)

Advirtamos que para Miró Quesada (1969) este principio y sus consecuencias pueden prescindir de las "tesis filosóficas de carácter teórico", y no necesitan ser probadas, pues son prescripciones y como tales "se aceptan o rechazan mediante actos de voluntad" (p. 108). "La proclamación de la dignidad de todos los hombres no es una teoría ni filosófica, ni científica, y no es posible tampoco probar que sea verdadera o falsa" (p. 30).

En lo esencial, sin duda, la primera propuesta en el campo de la práctica de FMQC coincide con lo que plantea Kant; sin embargo, nos interesa precisar tres diferencias entre los planteamientos y un punto más de coincidencia general, aunque la explicación difiera en su mayor parte.

Primero, cuando Kant (1980) se refiere a un fin, no se refiere a un telos por realizar, sino, más bien, a un "principio que es la condición suprema limitativa de la libertad de las 
acciones de todo hombre" (p. 46). Al respecto, Wood (1998) indica que hay que distinguir el concepto de un fin existente del concepto de fin como algo por realizar (p. 168). Además, este principio, que Kant califica de "principio objetivo" para diferenciarlo de cualquier principio proveniente de la experiencia (principio subjetivo), se origina en la razón pura.

En segundo lugar, Kant (1980) presenta, en el capítulo segundo de la Fundamentación de la metafísica de las costumbres, al principio como un postulado: "Ahora yo digo, el hombre, y en general todo ser racional, existe como fin en sí mismo", pero en el capítulo tercero expone su fundamentación, que se basa en la separación entre el mundo inteligible y el sensible: "El ser racional se considera como inteligencia perteneciente al mundo inteligible y si llama voluntad su causalidad es porque la considera solo como una causa eficiente que pertenece a ese mundo inteligible" (p. 60). El hombre como ser racional pertenece al mundo inteligible, y como ente sensible, al mundo fenoménico. El fenómeno está sometido a ciertas leyes, pero el ser racional en sí mismo está sometido a otras leyes. De ahí que cuando se juzgan las acciones se puede establecer cómo debieron ocurrir, aunque esto no haya ocurrido.

En tercer lugar, la propuesta de Kant sí es una teoría filosófica y pretende, en la medida que fundamenta su postulado, probar que es verdadera.

El punto extra de coincidencia general, pero que difiere en parte de su explicación, es que de los fines que son los seres racionales se sigue "la idea de la voluntad de todo ser racional como una voluntad universalmente legisladora" (Kant, 1980, p. 46), y este no es otro que el principio de la autonomía de la voluntad. El concepto del ser racional como legislador universal, a su vez, lleva al concepto de un reino de los fines: "el enlace sistemático de distintos seres racionales por leyes comunes" (p. 48). En este "todo tiene o un precio o una dignidad" (p. 48); la dignidad es algo con valor interno, a diferencia del precio, que admite el cambio o la sustitución. La autonomía se presenta, de este modo, como el fundamento de la dignidad humana.

Volvamos al último punto que habíamos tocado antes de hablar de las diferencias con Kant y que responde a la pregunta: ¿en qué se fundamenta el principio autotélico?, ya que se trata de un principio de una ideología estimativa; se basa en un acto de fe: "Sus principios [los de la ideología estimativa] se aceptan o no se aceptan, porque se cree o no se cree en ellos" (Miró Quesada, 1969, pp. 30-31).

Sin embargo, hay otra pregunta que cualquier estudioso de la filosofía se haría: ¿FMQC desconocía la propia explicación kantiana del ser racional como fin? ¡Por supuesto que no! Lo que posiblemente ocurrió es que esta primera moción (como se puede notar en nuestra introducción) es de los años sesenta del siglo xx y tiene como principal preocupación establecer una alternativa efectiva de cambio (que FMQC creía encontrar en Acción Popular), frente a las propuestas del aprismo y el comunismo. En esa situación, 
el esclarecimiento teórico detallado pasa a un segundo plano y se hace la referencia a la propuesta kantiana sin entrar en disquisiciones. Casi cuatro décadas después, Miró Quesada (2003a) sí hace puntuales deslindes con la propuesta kantiana:

Kant no logró superar el problema [enunciar un principio ético formal] porque la afirmación de que el hombre existe como un fin en sí es arbitraria. No hay ninguna razón que demuestre que el hombre es un fin en sí. (p. 205)

Podría pensarse que en este caso Miró Quesada (2003a) se refiere a la ética y no a la ideología (que se mueve, tal como lo ha señalado, en el campo de la política), pero él mismo indica que "la fundamentación racional de la ética constituye, asimismo, una fundamentación racional del derecho y la política" (p. 243). Pero volvamos a la crítica detallada contra Kant:

El intento kantiano de fundamentar racionalmente la ética no puede tener éxito por dos razones: (1) no logra establecer con claridad el concepto de forma de la ley moral, en tanto ella es el fundamento de todo comportamiento ético; (2) para superar el determinismo total de las leyes de la naturaleza, Kant llega al absurdo de suponer que la libertad solo puede ser de carácter noumenal. (p. 245)

Para terminar con esta sección, diremos que el principio autotélico se amplía y se convierte -en "Hombre, naturaleza, historia" (1987) - en proyecto autotélico. Con respecto a este trabajo, juzgado por Sobrevilla (1989) como "uno de los mejores ensayos filosóficos que se han escrito en el Perú" (p. 786), tomamos la siguiente observación:

a. En Miró Quesada el proyecto autotélico desemboca en un antropologismo que hace del hombre el centro del universo, en un antropocentrismo, posición que puede conducir a la pérdida del equilibrio entre el hombre y la naturaleza. (pp. 786-787)

Por supuesto, lo mismo se puede afirmar del principio autotélico: el fin es el hombre y esto va en detrimento ya no de otros (hombres), pero si esto se sobredimensiona, como ha ocurrido, va en perjuicio de la naturaleza y, por ende, del resto de las especies animales (Singer, 2009, pp. 65-71). FMQC es fiel al especismo humano proveniente de la tradición intelectual de la cultura occidental y al ideal baconiano.

\section{EL PRINCIPIO DE NO ARBITRARIEDAD}

A lo largo de su extensa obra, Miró Quesada (1969) se muestra como un decidido partidario de la racionalidad. A la razón la define como la facultad de convencimiento; así "hace posible la actividad suasoria de los hombres" (p. 147). El uso de esta facultad como criterio de decisión para tratar problemas teóricos y prácticos proviene de los filósofos griegos (Miró Quesada, 1992, pp. 203 y 220); pero ni siquiera entre los antiguos griegos el ideal de racionalidad llegó a convertirse en vigencia constitutiva. Vigencia es un concepto de Ortega y Gasset $(1983$, p. 222) que se refiere a una creencia que se 
encarna profundamente entre los hombres de un lugar o una época y que orienta tanto su forma de interpretar cierto tipo de fenómenos, así como las decisiones que toman frente a estos; por ejemplo, la idea del origen divino de la monarquía fue una vigencia incuestionable hasta el siglo XVII, pero actualmente ya no lo es.

Platón, en el libro VII de la República, es el primero en formular el ideal del conocimiento racional (509d-511e); este tiene las características de ser universal y necesario; pero, así como capta entes suprasensibles, también capta los principios supremos de la acción, es decir, lo que debemos hacer (519b-520d). De aquí, considera Miró Quesada (1969), se deriva el ideal de sociedad racional o sociedad justa. El ideal de vida racional empieza a cobrar vigencia en la Edad Media y a "mediados del siglo XVII se ha transformado en la vigencia fundamental" (p. 150). El florecimiento de esta transformación se produce con la llustración y "los enciclopedistas son los primeros en crear una ideología como justificación filosófica de la praxis política" (p. 151); pero justo cuando el enciclopedismo y el racionalismo llegan a su punto cumbre, Kant observa que el racionalismo que provenía de Descartes es insostenible en filosofía. Lo que vendría en los siglos XIx y xx sería más desastroso para la pretensión de una explicación suficiente desde el conocimiento racional, y esto ocurriría con la aparición de las geometrías no euclidianas, las paradojas de la teoría de los conjuntos, la crisis de la física clásica, la aparición de las lógicas no aristotélicas, etcétera (Miró Quesada, 1969, p. 153; 2003a, pp. 21-41).

Esta situación podría interpretarse como el fracaso de la razón o, como lo hace Miró Quesada (1969), “una depuración del sistema de la razón a través de un dinamismo constitutivo de la propia razón" (p. 156). En el ámbito práctico, lo que destaca FMQC es el papel cumplido por el análisis crítico racional que ha debilitado y cuestiona las vigencias que han sostenido a los regímenes de los grupos dominantes. La filosofía (racionalista) no ha podido establecer, sin embargo, "un sistema de principios evidentes para fundamentar la praxis política" (p. 163); y la experiencia histórica revela la escasa probabilidad de que este sistema logre encontrarse, pero esto no implica que no se pueda encontrar un cierto criterio de racionalidad. Es a partir de este señalamiento que Miró Quesada (1969) plantea el principio de no arbitrariedad: "Toda actividad racional presenta un aspecto negativo: la no arbitrariedad" (p. 163). Antes de volver sobre el principio citado, es pertinente leer una de las críticas de Sobrevilla (1989) sobre cómo FMQC concibe la racionalidad:

a. Para el filósofo peruano la racionalidad occidental es la racionalidad por excelencia: no repara en que otras culturas también han tenido otras formas de racionalidad [...]. En realidad, Miró Quesada tampoco distingue entre distintas formas de racionalidad en Occidente. La racionalidad del racionalismo moderno es para él la racionalidad a secas. (p. 787)

En cuanto al principio de no arbitrariedad (Miró Quesada, 1969), se presenta en el conocimiento racional o proceso puramente racional (racionalidad), cuyo poder radica 
en su capacidad de convencimiento, que deja sin efecto cualquier pretensión proveniente de una voluntad individual o grupal que se base en la ignorancia, el error, la apetencia, el interés o el capricho. "Por eso todo principio racional tiene siempre un aspecto negativo: es no arbitrario. La no arbitrariedad es el principio más general posible de la racionalidad" (p. 164). El concepto opuesto al de no arbitrariedad es el concepto de imposición, que se refiere a que una voluntad individual o grupal impone un curso de acción a una o más personas contra la voluntad de estas. De este segundo principio, se deriva una serie de consecuencias: la renuncia al uso de la violencia en los procesos de cambio social, el rechazo a toda forma de totalitarismo o dictadura, el afirmar como ideal la sociedad sin clases y la defensa de la democracia occidental.

De todas maneras, se presenta frente a este principio una paradoja, pues este segundo principio no acepta y, más bien, cuestiona las ideologías especulativas que consideran la necesidad inevitable del uso de la violencia para la transformación de la sociedad. El punto es que el uso de la violencia es por sí una actitud arbitraria. Entonces, "para luchar contra la arbitrariedad, hay que emplear la arbitrariedad. 0, lo que es lo mismo, para realizar la sociedad justa, hay que valerse de la injusticia" (Miró Quesada, 1969, p. 186). Pero el problema es de orden práctico y lo que ocurre es que no en toda situación revolucionaria se va a requerir usar la violencia; FMQC acepta que hay situaciones en las que el nivel de resistencia de los grupos dominantes hace inevitable el uso de la violencia, pero en estas la violencia debe ser temporal y vista como una desviación del principio que debe rectificarse en cuanto sea posible.

Con respecto a la democracia moderna, juzga que precisamente ha surgido con la intención de eliminar las arbitrariedades mediante el control de poder, así como de forjar una sociedad justa; aunque también señala que este intento de alcanzar el ideal aún se encuentra en su infancia. Pero la democracia moderna se presenta como la única alternativa viable que lleve a la humanidad hacia la sociedad justa.

A diferencia del principio autotélico, sobre el cual no vuelve FMQC, el principio de no arbitrariedad es revisado y modificado:

En el nivel teórico, la razón procede por principios, pero en el nivel práctico solo hay un principio sin ningún contenido [...]: el principio de la no arbitrariedad, es decir, el principio del consenso. A través del consenso universal, las normas éticas y jurídicas adquieren la universalidad y la necesidad que las hace racionales. La no arbitrariedad, el consenso de las voluntades, es condición necesaria y suficiente de la racionalidad de la praxis. (Miró Quesada, 1992, p. 230).

En este segundo momento, la no arbitrariedad es interpretada como consenso; Garzón (1992) considera este consenso - al que califica de fáctico- como un requisito que superaría el mero acto de fe propio de las ideologías estimativas; pero si tomamos al pie de la letra lo que escribe FMQC sobre la no arbitrariedad como condición necesaria 
y suficiente en el ámbito práctico, simplemente estaría dejando de lado la asunción de la ideología estimativa, o sea, el principio autotélico que se asumía como un acto de fe, y se estaría orientando hacia una propuesta de ideología racionalmente fundamentada; de ahí que ya no se vuelva a mencionar este principio y, más bien, se plantee como un proyecto a seguir.

En el "Ensayo de una fundamentación racional de la ética", se vuelve una vez más sobre el principio de no arbitrariedad, pero esta vez complementado por el principio de simetría, tomado de las ciencias físicas. Ahora este principio es presentado como condición suficiente y el principio de no arbitrariedad como condición necesaria, aunque los casos que se exponen solo se remiten al campo de la moralidad. Podría pensarse que tomar un principio que surge de teorías físicas es una extrapolación forzada, pero desde nuestro punto de vista es, más bien, la propuesta más audaz de Miró Quesada (2003a), pues al fin logra un principio formal que funciona tanto en el orden natural como en el campo de la práctica. Así, "la simetría y la no arbitrariedad constituyen las condiciones de validez tanto del conocimiento científico como del comportamiento ético" (p. 253).

La simetría también es un fenómeno que brota en Occidente con la primera constitución de Atenas y que va afirmándose y ampliándose al pasar por Roma, la Edad Media, el capitalismo; la estación final es la sociedad sin clases con "la igualdad total, esto es, la simetría de todas las relaciones colectivas y en especial las del poder" (Miró Quesada, 2003a, p. 259). FMQC es un progresista, pero a diferencia de un progresismo como el marxista (Marx y Engels, 1980, p. 55), que considera que el motor de la historia es la lucha de clases, Miró Quesada (2003a) ve el progreso como una creciente simetría en las relaciones entre los hombres en un mayor uso de su racionalidad, y la organización que mejor encarna este principio es la democracia moderna: "Desde el punto de vista político, la democracia es la expresión directa de la racionalidad, porque la esencia de la democracia consiste, precisamente, en la simetría del poder" (p. 242).

\section{CONCLUSIONES}

La propuesta ideológica de FMQC proviene de los años sesenta del siglo xx. De manera general, en su primera moción se complementan el principio autotélico, como principio positivo de acción y por el cual clasifica a su ideología como estimativa: respetar a los otros y hacer posible que se realicen como humanos; y el principio de no arbitrariedad, como principio negativo y de racionalidad: no aceptar la imposición, lo que va contra la voluntad y la libertad de los demás.

Posteriormente (1987), cambia a partir del momento en que considera al principio de no arbitrariedad como condición necesaria y suficiente para lo que compete al ámbito práctico. 
Esto también se modifica (2003a) cuando se introduce el principio de simetría, que, a semejanza del principio de no arbitrariedad, no se limita al campo práctico, y al cual presenta FMQC como complemento del principio de no arbitrariedad; ya no se refiere al principio de autotelia (que se plantea ahora como proyecto), lo cual implica que deja de lado el aspecto estimativo de su propuesta ideológica y más bien considera que ha logrado establecer la fundamentación formal que Kant no pudo lograr para el ámbito práctico.

\section{REFERENCIAS}

Garzón, E. (1992). Ideología y humanismo en la obra de Francisco Miró Quesada. En D. Sobrevilla y D. García Belaunde (Eds.), Lógica, razón y humanismo. La obra filosófica de Francisco Miró Quesada C. (pp. 199-218). Universidad de Lima, Fondo Editorial.

Kant, M. (1980). Fundamentación de la metafísica de las costumbres. Crítica de la razón práctica. La paz perpetua. Porrúa.

Marx, C., y Engels, F. (1980). Obras escogidas (t. 1). Progreso.

Miró Quesada, F. (1967). Manual ideológico. Primera parte. Acción Popular.

Miró Quesada, F. (1969). Humanismo y revolución. Casa de la Cultura del Perú.

Miró Quesada, F. (1992). Hombre, sociedad y política. Ariel, Comunicaciones para la Cultura. Miró Quesada, F. (2003a). Ser humano, naturaleza, historia. Paidós; Universidad Nacional Autónoma de México.

Miró Quesada, F. (2003b). El hombre, el mundo, el destino: introducción no convencional a la filosofía. Universidad de Lima, Fondo Editorial.

Ortega y Gasset, J. (1983). Obras completas (t. VII). Alianza Editorial; Revista de Occidente.

Platón. (1992). Diálogos IV. República. Gredos.

Popper, K. (1980). La lógica de la investigación científica. Tecnos.

Singer, P. (2009). Ética práctica. Akal.

Sobrevilla, D. (1989). Repensando la tradición nacional I (vol. 2). Hipatia.

Wood, A. (1998). Humanity as End in Itself. En P. Guyer (Ed.), Kant's Groundwork of the Metaphysics of Morals: Critical Essays (pp. 165-188). Rowman \& Littlefield Publishers. 